\title{
Differences in responding for sucrose and wheel-running reinforcement: Excitatory stimulus effects or inhibitory after-effects?
}

\author{
TERRY W. BELKE \\ Mount Allison University, Sackville, New Brunswick, Canada
}

\begin{abstract}
Previous research showed that sucrose and wheel-running reinforcement of leverpressing generate different response rate asymptotes. To investigate the basis of this difference, the present study assessed the role of inhibitory after-effects and excitatory stimulus effects on measures of responding in rats exposed to fixed-interval schedules that randomly produced either sucrose or wheel-running reinforcers. Different discriminative stimuli were associated with each reinforcer type. Inhibitory aftereffects and excitatory stimulus effects were assessed by the pattern of postreinforcement pauses and local response rates across the four different combinations of previous and upcoming reinforcer types: wheel-wheel, wheel-sucrose, sucrose-wheel, and sucrose-sucrose. Results showed that, regardless of the prior type of reinforcer, response rates were higher and pauses were shorter in the presence of a stimulus signaling sucrose reinforcement. This suggests that differences in response rate asymptotes generated by these qualitatively different reinforcers may have more to do with differences in excitatory stimulus effects than with inhibitory after-effects.
\end{abstract}

In 1970, Herrnstein formulated a form of the matching law equation for the situation in which there is only a single source of reinforcement arranged by the experimenter and a single measured response. In this single-operant form of the matching law, the response-reinforcer relation takes the form of a hyperbola that is described by the following equation:

$$
B 1=\frac{k R 1}{R 1+R e},
$$

where $B 1$ is the predicted response rate (responses $/ \mathrm{min}$ ), $R 1$ is the obtained reinforcement rate, and $k$ and $R e$ are estimated parameters. Specifically, $k$ refers to the asymptotic response rate and $R e$ is defined by the rate of reinforcement that maintains half the asymptotic response rate.

In 1998, Belke showed that response rate asymptotes in rats responding on a lever for $.1 \mathrm{ml}$ of $15 \%$ sucrose solution were higher than those obtained when the opportunity to run for $15 \mathrm{sec}$ was the reinforcing consequence. This finding contradicts a major assumption underlying Herrnstein's (1970) single-operant form of the matching law. According to Herrnstein (1974), "The sole determining influence on the size of [the response rate asymptote] is the response form itself, without regard to the amount or type of reinforcement conditional upon it or on anything else" (p. 163). In other words, response rate asymptotes for sucrose and wheel-running reinforcement should

This research was supported by Grant 0GP0170022 from the Natural Sciences and Engineering Research Council of Canada. Correspondence should be sent to T. W. Belke, Department of Psychology, Mount Allison University, Sackville, NB, E4L 1C7 Canada (e-mail: tbelke@) mta.ca). not differ unless the form of a response that each reinforcer is contingent upon differs.

More recently, studies using sucrose and water reinforcement produced results that violate this assumption. Dallery, McDowell, and Lancaster (2000) showed that response rate asymptotes within the same animal were relatively constant across sucrose concentrations ranging from 0.64 to $0.2 \mathrm{M}$, but decreased at the concentrations of 0.1 and 0.05 M. McDowell and Dallery (1999) showed that for rats pressing levers for water reinforcement, response rate asymptotes remained relatively constant between 23.5 and $6 \mathrm{~h}$ of deprivation, but declined markedly with deprivation levels of 4 and $2 \mathrm{~h}$. According to the researchers, the difference between these studies and previous studies that failed to show any violation of Herrnstein's assumption (several researchers analyzed results from studies prior to 1976: Bradshaw, Ruddle, \& Szabadi, 1981; Bradshaw, Szabadi, Ruddle, \& Pears, 1983; Conrad \& Sidman, 1956; Guttman, 1954; Heyman \& Monaghan, 1987, 1994; Kraeling, 1961; de Villiers \& Herrnstein, 1976 , analyzed results from studies prior to 1976 ) is that the independent variable (i.e., sucrose concentration, hours of deprivation) was manipulated over a greater range of values.

If this is the case, then the difference in response rate asymptotes between sucrose and wheel-running reinforcement may be equivalent to the difference between a high and a low concentration of sucrose. Consequently, the difference in response rate asymptotes would be predicted to disappear if the concentration of sucrose reinforcement was markedly diminished. The decrease in sucrose concentration would diminish the efficacy of the reinforcer for generating and maintaining responding. 
Alternatively, the difference in the asymptotes may be a function of postreinforcement inhibitory effects. Running and drinking sucrose solution may generate qualitatively different inhibitory after-effects. Inhibitory aftereffects following the consumption of sucrose may be a function of central satiation effects. For wheel running, after-effects may be a function of either central satiation or peripheral muscular fatigue effects. If the inhibitory after-effect following a period of wheel running is greater than that following the consumption of a drop of sucrose solution, this difference may be reflected in the response rate asymptotes generated by these reinforcers.

To assess the relative contribution of inhibitory aftereffects and excitatory stimulus effects associated with these qualitatively different reinforcers, the present study used a procedure similar to one developed by Perone and Courtney (1992). In their study, pigeons responded on fixed-ratio (FR) schedules with reinforcers of different magnitudes delivered when the schedule requirement was met. Each reinforcer magnitude was signaled by a different stimulus. Patterns of pausing were assessed across a series of transitions defined by the magnitude of the previous reinforcer and the magnitude of the upcoming reinforcer. Larger magnitude reinforcers were assumed to have greater excitatory stimulus effects and inhibitory after-effects. Pauses were found to be a function of both the previous reinforcer and the stimulus correlated with the upcoming reinforcer.

In the present study, rats responded on fixed-interval (FI) schedules for either wheel-running or sucrose reinforcement. Each type of reinforcer was signaled by a different stimulus. Transitions defined by the previous reinforcer type and the upcoming reinforcer type corresponded to sucrose followed by sucrose, sucrose followed by wheel, wheel followed by sucrose, and wheel followed by wheel. Depending upon the assumptions made about relative inhibitory and excitatory effects, different patterns would be predicted for pause durations and response rates across these four different transitions. For example, if wheel-running and sucrose reinforcement differ in inhibitory after-effects, but not excitatory stimulus, and if the inhibitory after-effect of wheel running is greater than that of sucrose, then one would expect longer pauses for wheel-wheel and wheel-sucrose transitions and shorter pauses for sucrose-sucrose and sucrose-wheel transitions. Alternatively, if wheel running and sucrose are assumed to differ in excitatory stimulus effects, but not inhibitory after-effects, and if the excitatory effects of sucrose are assumed to be greater, then one would expect shorter pauses for the sucrose-sucrose and wheelsucrose transitions and longer pauses for the wheelwheel and sucrose-wheel transitions. In addition, the greater excitatory effect of sucrose would produce a higher rate of responding in the presence of the stimulus signaling sucrose. Finally, if wheel running was assumed to have a greater inhibitory after-effects and sucrose as- sumed to have greater excitatory effects, then one would expect the longest pauses for wheel-wheel transitions and the shortest pauses for sucrose-sucrose transitions. In the former case, the greater inhibitory after-effect and weaker excitatory effect of wheel-running reinforcement would combine to produce longer pause durations. In the latter case, the weaker inhibitory after-effect and greater excitatory effect would combine to produce shorter pause durations. Pause durations associated with the remaining two transitions, wheel-sucrose and sucrose--wheel, would fall between the other two transitions. Thus, different sets of assumptions regarding relative excitatory effects and inhibitory after-effects would predict different patterns of pausing across the four transitions.

\section{METHOD}

\section{Subjects}

Six male Wistar rats obtained from Charles River Breeding Laboratories (Quebec) served as subjects. All animals were approximately 7 months old at the start of the experiment. The rats were individually housed in polycarbonate cages $(48 \times 27 \times 22 \mathrm{~cm})$ in a holding room on a 12:12-h light:dark cycle (lights on 0800 ). Immediately after each experimental session, each rat was given an amount of food sufficient to maintain its weight at approximately $85 \%$ of a free-feeding body weight determined when each animal had reached an adult weight of approximately $400 \mathrm{~g}$. Target weights varied from 330 to $340 \mathrm{~g}$. Distilled water was freely available in the home cage.

Also, data from the 4 rats that demonstrated higher response rate asymptotes for sucrose in Belke's (1998) study were included in the present study. These 4 animals were maintained under identical conditions and exposed to an experimental procedure similar to that used in this study, but at an earlier date.

\section{Apparatus}

Sessions occurred in two activity wheels (Lafayette Instruments) with diameters of $35 \mathrm{~cm}$. Each wheel was located in a soundattenuating shell with a fan to afford ventilation and to mask extraneous noise. Wheel revolutions were recorded by a microswitch attached to the wheel frame, and $24 \mathrm{~V} \mathrm{DC}$ lights mounted on the sides of the wheel frame illuminated the interior of the wheel chamber. A solenoid-operated brake was attached to the base of the wheel frame. When the solenoid was operated, a rubber tip attached to a metal shaft contacted the wheel and caused the wheel to stop.

A Plexiglas panel $(16 \times 16.5 \times 0.4 \mathrm{~cm})$ with a lever, two stimulus lights, and a liquid receptacle was mounted at the opening of each wheel $(7 \times 9 \mathrm{~cm})$. The lever was located $10 \mathrm{~cm}$ from the base of each panel. Each lever was $3.3 \mathrm{~cm}$ wide and extended $2 \mathrm{~cm}$ from the face of the panel into the wheel chamber. The force required to activate the lever microswitch in each wheel was approximately $30 \mathrm{~g}$. Located $1.25 \mathrm{~cm}$ above each lever were red and white $28 \mathrm{~V} \mathrm{DC}$ $40 \mathrm{~mA}$ stimulus lights (Dialco 507-3917). The diameter of each light was $7 \mathrm{~mm}$, and the center-to-center distance between the two lights was $1.4 \mathrm{~cm}$. The white stimulus light was located to the left of the red stimulus light in one wheel and to the right in the other. Immediately adjacent to each lever was a liquid receptacle. The area of each receptacle into which sucrose solution was dispensed was $5.5 \times 6 \times 3.2 \mathrm{~cm}$. The base of each receptacle was located $5.7 \mathrm{~cm}$ from the base of each panel. Behind the top of each receptacle was a metal clamp into which a clear plastic cylinder $(10.5 \mathrm{~cm}$ long, $3.8 \mathrm{~cm}$ diameter) and a 24 V DC General Valve Co. solenoid could be 
Table 1

Order of Stimulus Conditions for Each Rat Responding on a Tandem FR1 VI 30-sec Schedule Denoted by Illumination, Color, and Reinforcer Type

\begin{tabular}{lllll}
\hline & & \multicolumn{2}{c}{ Condition } & \multicolumn{1}{c}{ 1 } \\
\cline { 2 - 4 } Rat & \multicolumn{1}{c}{2} & \multicolumn{1}{c}{3} \\
H4 & Blink Red Sucrose & Solid Red Sucrose & Blink White Wheel & Solid White Wheel \\
H9 & Solid White Sucrose & Blink White Sucrose & Solid Red Wheel & Blink Red Wheel \\
H11 & Blink White Wheel & Solid White Wheel & Blink Red Sucrose & Solid Red Sucrose \\
H12 & Solid Red Wheel & Blink Red Wheel & Solid White Sucrose & Blink White Sucrose \\
H3 & Blink Red Sucrose & Blink White Wheel & Solid Red Sucrose & Solid White Wheel \\
H8 & Solid Red Wheel & Solid White Sucrose & Blink Red Wheel & Blink White Sucrose
\end{tabular}

Note-In blink conditions, the stimulus light was briefly extinguished with each leverpress; in solid conditions, the stimulus light was extinguished only when the reinforcer was delivered.

placed. A Lafayette Instruments Co. Model 80201 liquid dispenser operated the solenoid valve. Each Plexiglas panel was attached to the wheel frame by Velcro strips. Control of experimental events and recording of data was handled by a Borland Turbo Pascal 4.0 program run on an IBM PC computer interfaced to the wheel through the parallel port.

\section{Procedure}

Initially, 17 rats were given free access to a running wheel for $30 \mathrm{~min}$ each day for 10 days. The number of wheel revolutions was recorded for each rat on each day. After 10 days, the 6 highest rate runners were selected for the present study. In the next phase the rats continued to receive $30-\mathrm{min}$ access to the free-moving running wheel. In addition, each rat was placed in an ordinary operant conditioning chamber, and leverpressing was shaped by the method of reinforcing successively closer approximations. Each leverpress produced $0.1 \mathrm{ml}$ of a $15 \%$ sucrose solution. When subjects reliably pressed the lever, the schedule of reinforcement was shifted from requiring only a single response per reinforcer (FR 1) to one requiring a variable number of responses averaging 3 (i.e., a variableratio [VR] schedule). This schedule remained in effect for approximately four sessions, with each session terminating when 50 sucrose reinforcers had been obtained.

After four sessions on the VR 3 schedule, sessions in the operant conditioning chamber were discontinued. At this point, the retractable lever in each wheel chamber was extended during the wheel-running sessions and the opportunity to run for $60 \mathrm{sec}$ was made contingent upon a single leverpress. Retraction of the lever and movement of the wheel with the release of the brake signaled access to the running period. Each session consisted of 30 opportunities to run. The schedule of reinforcement was changed in the following sequence: FR 1, VR 3, VR 5, VR 9, and VR 15. Subjects remained on each schedule for four sessions before advancing to the next schedule.

In preparation for the present study, animals trained with the procedure described above were exposed to each type of reinforcement separately in the presence of different stimuli. Since Wistar rats are unlikely to discriminate differences in color, the discriminability of the two stimuli was increased by having one of the stimulus lights "blink" off when the lever was pressed and the other remain on when the lever was pressed. That is, the light that was made to blink would be extinguished for $0.5 \mathrm{sec}$ following the occurrence of a leverpress. Blinking was associated with different lights for different rats. Since the offset of the stimulus light was also associated with the delivery of the reinforcer, blinking could function as a conditioned reinforcer; therefore, it was necessary that the blinking be associated with each reinforcement type. Exposing the animals to these initial conditions would allow for the assessment of the conditioned reinforcement effect of blinking, if any. Table 1 shows the order of presentation of these initial conditions. The schedule of re- inforcement during this phase was a tandem FR 1 variable-interval (VI) 30-sec schedule. Each session terminated when 50 reinforcements had been obtained. Each condition in this initial phase remained in effect for 25 sessions.

Following this initial phase, the animals were exposed to a standard FI 30-sec schedule of reinforcement with either the opportunity to run for $15 \mathrm{sec}$ or $0.1 \mathrm{ml}$ of a $15 \%$ sucrose solution as the reinforcing consequence. A different stimulus light was associated with each of these consequences. Stimulus light assignments corresponded to the stimuli that the animals were exposed to in Conditions 2 and 3 in Table 1. For example, for Rat H4, sucrose was signaled by a red stimulus light and wheel running was signaled by a white stimulus light that was extinguished briefly when a leverpress occurred. The type of reinforcement that occurred on any given FI period was determined by random selection from a set of two values. When the reinforcement requirement was met, the stimulus light signaling a wheel-running reinforcer was extinguished for the duration of the reinforcement period, while the stimulus signaling sucrose reinforcement was extinguished for $0.5 \mathrm{sec}$. Each session consisted of 100 reinforcements. This condition remained in effect for 100 sessions. Over this period, all rats developed stable differentiation of responding in the presence of the stimuli signaling the different reinforcers.

Leverpresses, time spent leverpressing, postreinforcement pause (PRP), and wheel revolutions (for wheel-running reinforcement only) were recorded for each reinforcer and cumulatively for the entire session. Wheel-running rates were calculated as the total number of revolutions per session divided by the total time during which running could occur and expressed as revolutions $/ \mathrm{min}$. A local leverpressing rate was also calculated. This rate was calculated as total leverpresses divided by total time spent pressing the lever, exclusive of the PRP time, and expressed as presses $/ \mathrm{min}$. Median PRPs were obtained for the distribution of pauses over the last five sessions. Leverpresses during successive 5-sec segments of the 30 -sec reinforcement interval were cumulated over the entire session separately for intervals that terminated in wheel-running and sucrose reinforcement. Finally, wheel revolutions during successive 5-sec segments of the 15-sec reinforcement period were cumulated across all wheel-running reinforcers within a session.

The 4 rats from Belke's (1998) study were exposed to a similar procedure. Notable differences were that a single running wheel was used to run the procedure, a "blinking" red stimulus light was associated with sucrose reinforcement, a solid white stimulus light was associated with wheel-running reinforcement, and the duration of the wheel-running reinforcer was varied. Of these exceptions, failure to counterbalance "blinking" across reinforcers is the most critical. The offset of the stimulus light was associated with both a leverpress and the delivery of sucrose solution. This raised the possibility that a higher rate of responding in the presence of the stimulus signaling sucrose solution reflected a conditioned reinforce- 
ment effect rather than a difference in reinforcer efficacy. This issue was addressed by comparison of response rates across the conditions presented in Table 1 .

\section{RESULTS}

Figure 1 shows local leverpressing rates for each type of reinforcer and stimulus condition for each rat from the last five sessions of initial phase in which the animals were responding on tandem FR 1 VI 30-sec schedules. Visual inspection of Figure 1 shows that local leverpressing rates were systematically higher for sucrose than for wheel-running reinforcement; however, no systematic differences within each reinforcer type as a function of stimulus condition are apparent. Mean response rates for the sucrose blink, sucrose solid, wheel blink, and wheel solid conditions were $54.57,52.41,26.66$, and $28.33 \mathrm{presses} / \mathrm{min}$, respectively. A repeated measures analysis of variance (ANOVA) with reinforcer type (sucrose, wheel) and stimulus condition (blink, solid) as within-subjects variables revealed a significant effect of reinforcer type $[F(1,5)=69.40, p<.001]$, but no effect of stimulus condition $[F(1,5)=0.02, p>.10]$, and no reinforcer type $\times$ stimulus condition interaction $[F(1,5)=$ $1.15, p>.10]$. The lack of an effect of stimulus condition suggests that extinguishing the stimulus light when the animal pressed the lever did not function as a conditioned reinforcer to increase responding.

Figure 2 depicts median PRPs for the distribution of pauses over the last five sessions of the same initial phase for each rat. Readily apparent is the observation that median PRPs following wheel reinforcers were longer than those following sucrose reinforcers. Average median pauses for the sucrose blink, sucrose solid, wheel blink, and wheel solid conditions were 5.82, 5.56, 17.14, and $16.96 \mathrm{sec}$, respectively. A repeated measures ANOVA revealed a significant effect of reinforcer type $[F(1,5)=$ $27.30, p<.01]$, but no effect of stimulus condition $[F(1,5)=0.15, p>.10]$, and no reinforcer type $\times$ stimulus condition interaction $[F(1,5)=0.01, p>.10]$. As was the case for local leverpressing rates, these data do not support that extinguishing the stimulus light when the animal pressed the lever functioned as a conditioned reinforcer. On the basis of these analyses of response rates and PRPs on tandem FR1 VI schedules, data from the 4 rats that had previously demonstrated differences in response rate asymptotes for wheel running and sucrose were incorporated into all subsequent analyses.

Figure 3 depicts mean responses in successive 5-sec bins of the FI $30-\mathrm{sec}$ reinforcement interval in the presence of stimuli signaling wheel-running and sucrose reinforcers for the first and last five sessions. For the $\mathrm{H}$-series rats, the last five sessions were Sessions 95-100; for the $\mathrm{M}$-series rats, the last five sessions were Sessions 171175. The difference in sessions between the two series reflects the additional sessions during which the rein- forcer duration for the M-series rats was changed from 15 to $30 \mathrm{sec}$ and then returned to $15 \mathrm{sec}$. The last five sessions of the second exposure to a wheel-running reinforcer duration of $15 \mathrm{sec}$ are depicted in Figure 3.

Visual inspection of this figure shows that with exposure to the procedure, each rat developed patterns of responding typical of FI schedules and differential rates of responding in the presence of stimuli signaling different reinforcer types. Between the first and last five sessions, all rats developed patterns of responding indicative of temporal discrimination of the reinforcement interval regardless of reinforcer type. That is, in the presence of stimuli signaling both reinforcer types, responding immediately following the termination of the reinforcer was low, and as the interval elapsed, the rate of responding increased. In contrast, during the first five sessions, responding in the presence of either stimulus was low immediately following the termination of the reinforcer and then rapidly rose to a higher level with little change across successive 5 -sec bins.

More importantly, in addition to this change in response pattern, which is indicative of temporal discrimination, differential responding in the presence of stimuli signaling reinforcer type also developed. For the first five sessions, responding in the presence of the stimuli signaling sucrose and wheel-running reinforcement did not differ. However, by the last five sessions, response rate functions in the presence of the stimulus signaling sucrose reinforcement rose more quickly to higher levels than did those in the presence of the stimulus signaling wheelrunning reinforcement.

Table 2 shows the breakdown of transitions by previous and upcoming reinforcer type for each rat over the last five sessions. Because transitions were generated by random selection rather than preset sequences, each type did not occur equally often within a session and varied across sessions. Across all animals, mean wheel-wheel, wheel-sucrose, sucrose-wheel, and sucrose-sucrose transitions were $24.06,24.44,24.40$, and 26.10 . These values suggest that over the last five sessions, wheel-wheel transitions occurred less often than expected (24.75) and sucrose-sucrose transitions more often. Although the deviations are not substantial and could be diminished by averaging over a greater number of sessions, their potential effect is unknown. Despite this uncertainty, data from these sessions were used to assess patterns of local leverpressing rates and PRPs across transitions.

To obtain local leverpressing rates, a response rate was calculated for each reinforcer by dividing the number of responses that occurred between the first response and the response that produced the reinforcement by the time between these two responses. Since these response rates were to be sorted by category of previous and upcoming reinforcer, the first reinforcer from each session was eliminated. In addition, any reinforcer where the first response was also the response that produced reinforce- 


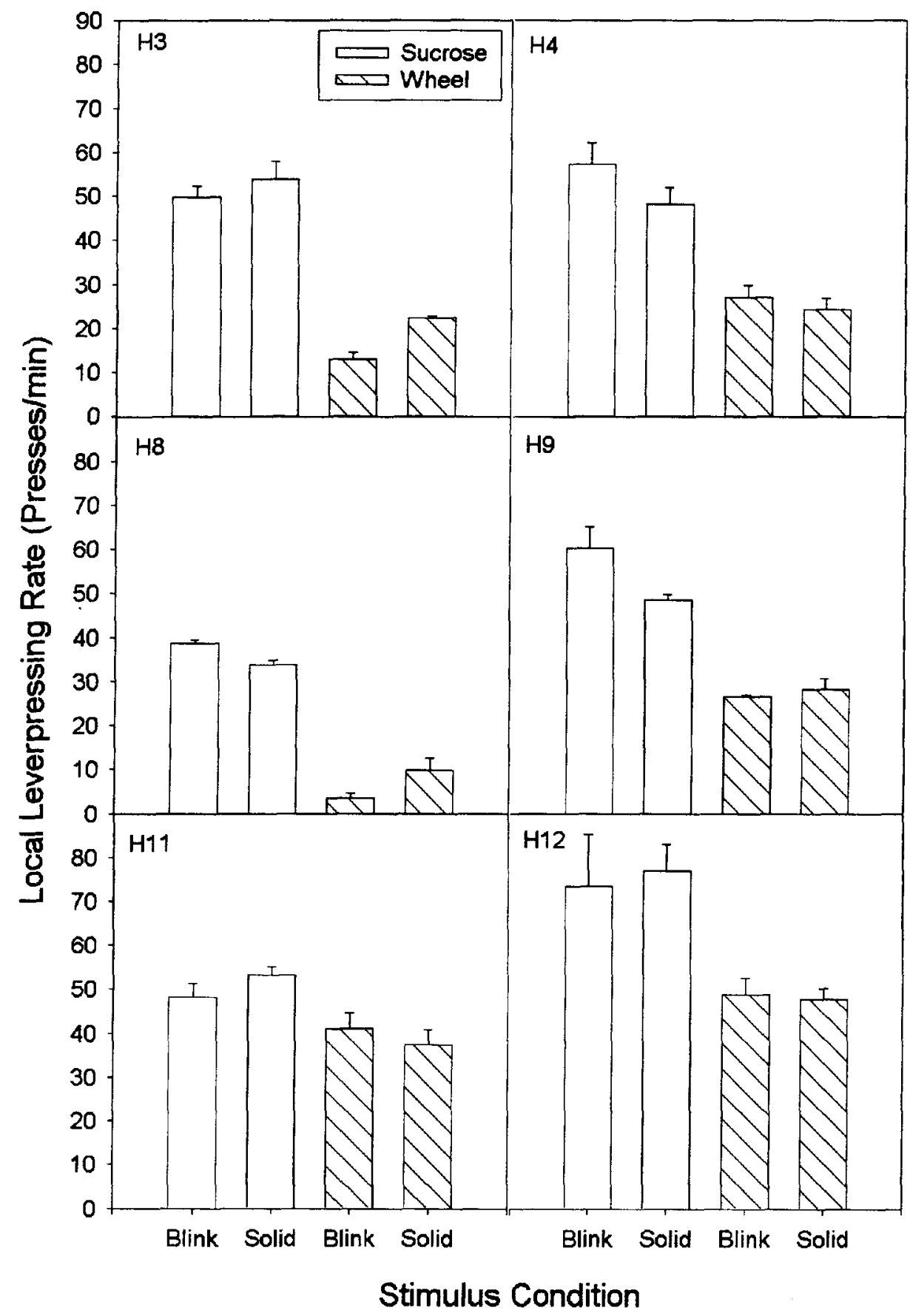

Figure 1. Local leverpressing rates (presses/min) on tandem FR 1 VI 30-sec schedules for wheelrunning and sucrose reinforcement for each animal as a function of stimulus condition. In the blink stimulus condition, the stimulus light above the lever was extinguished briefly when the lever was pressed; in the solid condition, leverpresses had no effect on the stimulus light. In both stimulus conditions, the stimulus light was extinguished when the reinforcer was delivered. Standard deviations are depicted for each mean.

ment because the PRP was longer than the schedule requirement was also eliminated because no response rate could be calculated for these reinforcers.

Table 3 shows the breakdown of reinforcers by reinforcer type over the last five sessions. In addition, the total number of reinforcers obtained with a single lever is broken down by reinforcer type. With the exception of Rat H3, the number of PRPs longer than $30 \mathrm{sec}$ in the presence of the stimulus signaling a wheel-running reinforcer was greater than that in the presence of the stim- 


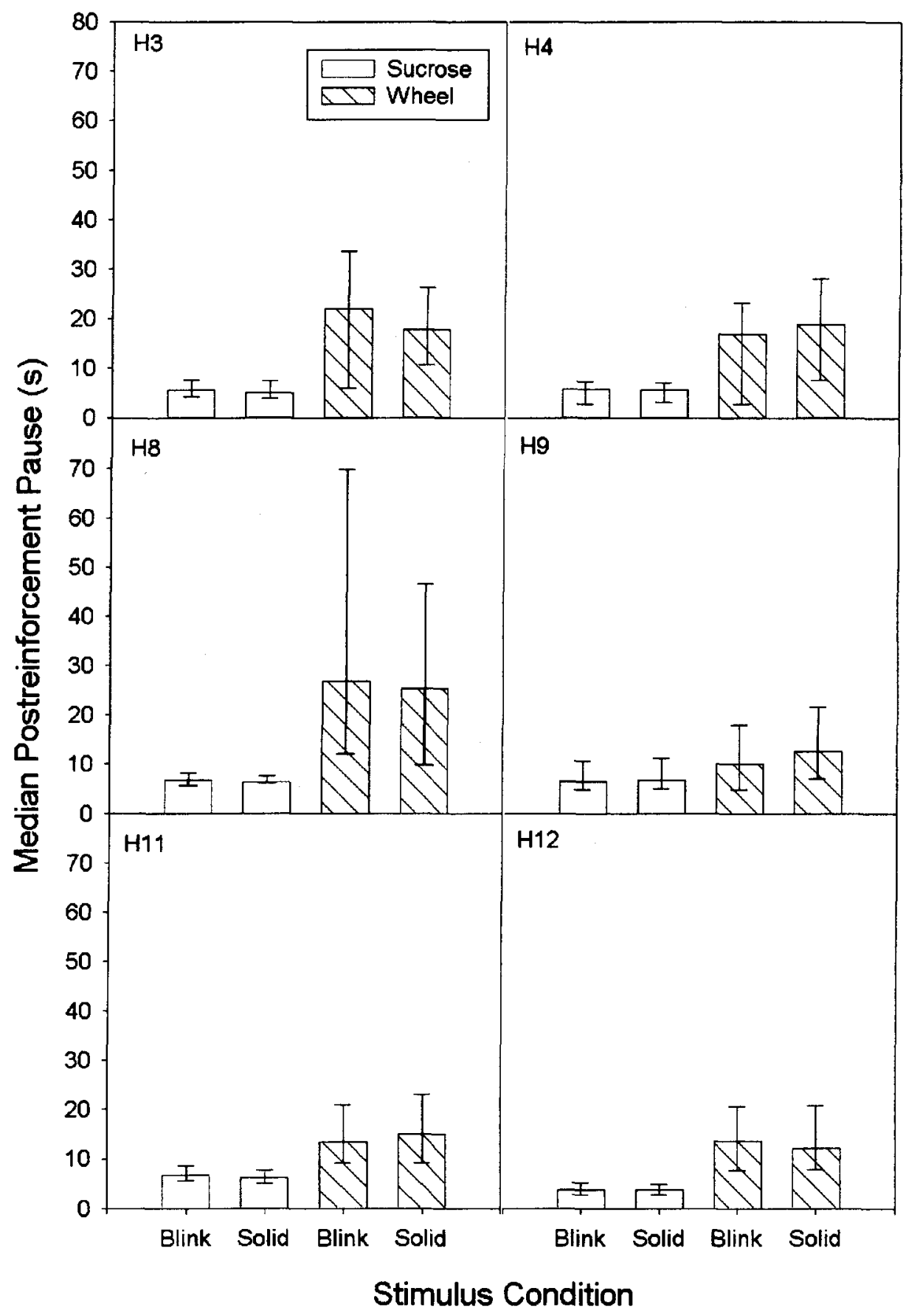

Figure 2. Median postreinforcement pause duration (sec) on tandem FR 1 VI 30-sec schedules for wheel-running and sucrose reinforcement for each animal as a function of stimulus condition. In the blink stimulus condition, the stimulus light above the lever was extinguished briefly when the lever was pressed; in the solid condition, leverpresses had no effect on the stimulus light. In both stimulus conditions, the stimulus light was extinguished when the reinforcer was delivered. Interquartile ranges are depicted for each median.

ulus signaling sucrose reinforcement. Rat $\mathrm{H} 3$ had slightly more PRPs longer than $30 \mathrm{sec}$ in the presence of the stimulus signaling sucrose reinforcement. On average, 28 out of 246 reinforcers in the presence of the wheel stimulus had PRPs longer than $30 \mathrm{sec}$ compared with 4 out of 254 in the presence of the sucrose stimulus. A paired $t$-test comparison showed that PRPs longer than the schedule requirement were more likely to occur in the presence of a stimulus signaling a wheel-running reinforcer $[t(9)=2.95$, $p<.05]$. 


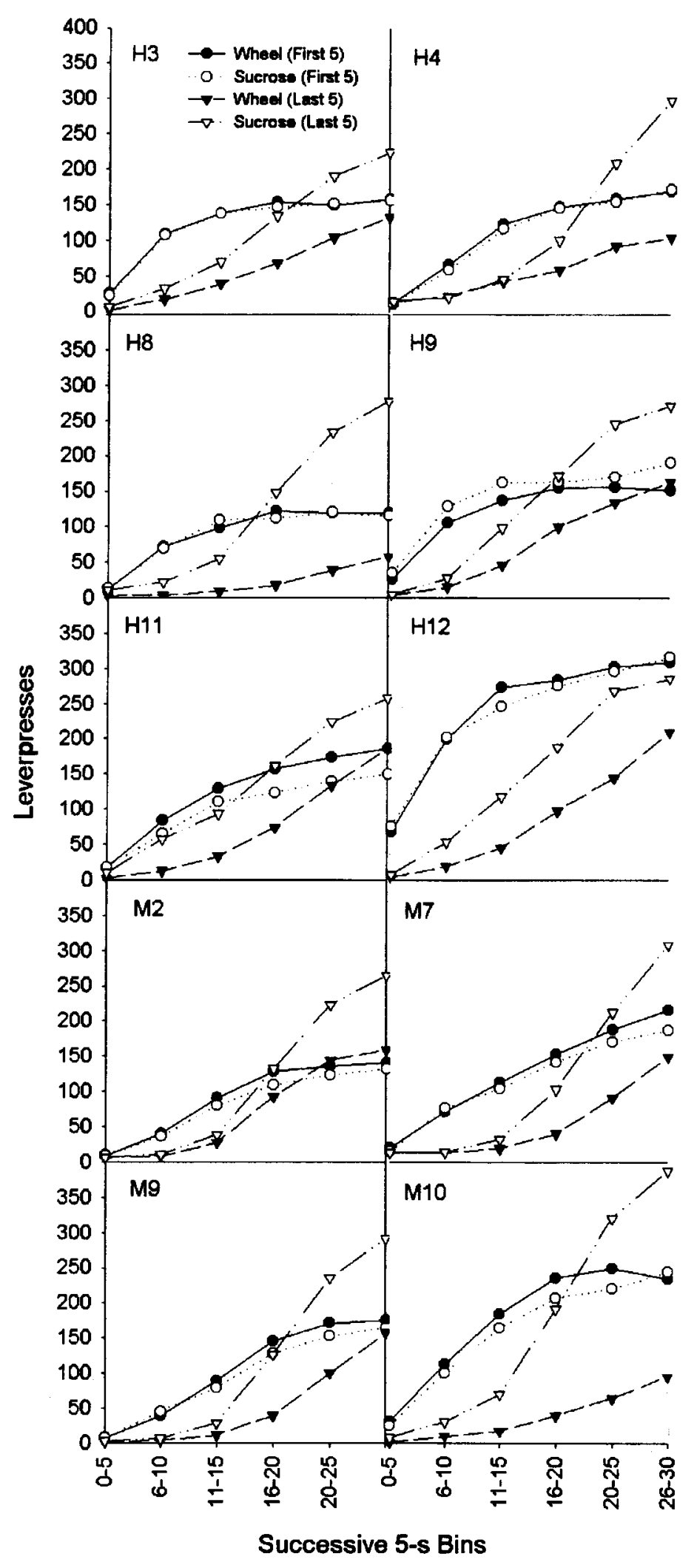

Figure 3. Mean responses in successive 5-sec segments of an FI 30-sec reinforcement interval in the presence of stimuli signaling wheel-running and sucrose reinforcers for the first and last five sessions. Data from the 4 animals previously exposed to this procedure (M2 to M10) are included. 
Table 2

Transitions Defined by Previous and Upcoming Reinforcer Type for Each of the Last Five Sessions for Each Animal

\begin{tabular}{|c|c|c|c|c|c|}
\hline Rat & Session & Wheel-Wheel & Wheel-Sucrose & Sucrose-Wheel & Sucrose-Sucrose \\
\hline $\mathrm{H3}$ & $\begin{array}{l}1 \\
2 \\
3 \\
4 \\
5\end{array}$ & $\begin{array}{l}24 \\
25 \\
27 \\
28 \\
29\end{array}$ & $\begin{array}{l}24 \\
23 \\
21 \\
21 \\
21\end{array}$ & $\begin{array}{l}25 \\
23 \\
21 \\
21 \\
21\end{array}$ & $\begin{array}{l}26 \\
28 \\
30 \\
29 \\
28\end{array}$ \\
\hline$M$ & & $26.6(1.85)$ & $22.0(1.26)$ & $22.2(1.60)$ & $28.2(1.33)$ \\
\hline $\mathrm{H} 4$ & $\begin{array}{l}1 \\
2 \\
3 \\
4 \\
5\end{array}$ & $\begin{array}{l}19 \\
20 \\
24 \\
27 \\
17\end{array}$ & $\begin{array}{l}27 \\
26 \\
23 \\
23 \\
25\end{array}$ & $\begin{array}{l}26 \\
26 \\
23 \\
22 \\
25\end{array}$ & $\begin{array}{l}27 \\
27 \\
29 \\
27 \\
32\end{array}$ \\
\hline$M$ & & $21.4(3.61)$ & $24.8(1.60)$ & $24.4(1.62)$ & $28.4(1.96)$ \\
\hline $\mathrm{H} 8$ & $\begin{array}{l}1 \\
2 \\
3 \\
4 \\
5\end{array}$ & $\begin{array}{l}27 \\
29 \\
21 \\
17 \\
19\end{array}$ & $\begin{array}{l}27 \\
25 \\
27 \\
26 \\
23\end{array}$ & $\begin{array}{l}26 \\
25 \\
27 \\
25 \\
22\end{array}$ & $\begin{array}{l}19 \\
20 \\
24 \\
31 \\
35\end{array}$ \\
\hline$M$ & & $22.6(4.63)$ & $25.6(1.50)$ & $25.0(1.67)$ & $25.8(6.24)$ \\
\hline H9 & $\begin{array}{l}1 \\
2 \\
3 \\
4 \\
5\end{array}$ & $\begin{array}{l}19 \\
22 \\
30 \\
28 \\
25\end{array}$ & $\begin{array}{l}25 \\
24 \\
23 \\
23 \\
23\end{array}$ & $\begin{array}{l}25 \\
25 \\
23 \\
24 \\
22\end{array}$ & $\begin{array}{l}30 \\
28 \\
23 \\
24 \\
29\end{array}$ \\
\hline$M$ & & $24.8(3.97)$ & $23.6(0.80)$ & $23.8(1.17)$ & $26.8(2.79)$ \\
\hline HII & $\begin{array}{l}1 \\
2 \\
3 \\
4 \\
5\end{array}$ & $\begin{array}{l}31 \\
23 \\
20 \\
27 \\
19\end{array}$ & $\begin{array}{l}23 \\
24 \\
24 \\
26 \\
29\end{array}$ & $\begin{array}{l}24 \\
25 \\
23 \\
26 \\
28\end{array}$ & $\begin{array}{l}21 \\
27 \\
32 \\
20 \\
23\end{array}$ \\
\hline$M$ & & $24.0(4.47)$ & $25.2(2.14)$ & $25.2(1.72)$ & $24.6(4.41)$ \\
\hline $\mathrm{H} 12$ & $\begin{array}{l}1 \\
2 \\
3 \\
4 \\
5\end{array}$ & $\begin{array}{l}21 \\
21 \\
26 \\
22 \\
23\end{array}$ & $\begin{array}{l}26 \\
27 \\
23 \\
28 \\
22\end{array}$ & $\begin{array}{l}26 \\
28 \\
23 \\
27 \\
22\end{array}$ & $\begin{array}{l}26 \\
23 \\
27 \\
22 \\
32\end{array}$ \\
\hline$M$ & & $22.6(1.85)$ & $25.2(2.32)$ & $25.2(2.32)$ & $26.0(3.82)$ \\
\hline M2 & $\begin{array}{l}1 \\
2 \\
3 \\
4 \\
5\end{array}$ & $\begin{array}{l}23 \\
25 \\
21 \\
26 \\
20\end{array}$ & $\begin{array}{l}25 \\
23 \\
25 \\
22 \\
27\end{array}$ & $\begin{array}{l}25 \\
23 \\
26 \\
23 \\
28\end{array}$ & $\begin{array}{l}26 \\
28 \\
27 \\
28 \\
24\end{array}$ \\
\hline$M$ & & $23.0(2.28)$ & $24.4(1.74)$ & $25.0(1.90)$ & $26.6(1.50)$ \\
\hline M7 & $\begin{array}{l}1 \\
2 \\
3 \\
4 \\
5\end{array}$ & $\begin{array}{l}19 \\
19 \\
21 \\
30 \\
27\end{array}$ & $\begin{array}{l}28 \\
25 \\
28 \\
21 \\
24\end{array}$ & $\begin{array}{l}27 \\
25 \\
29 \\
21 \\
24\end{array}$ & $\begin{array}{l}25 \\
30 \\
21 \\
27 \\
24\end{array}$ \\
\hline$M$ & & $23.2(4.49)$ & $25.2(2.64)$ & $25.2(2.71)$ & $25.4(3.01)$ \\
\hline M9 & $\begin{array}{l}1 \\
2 \\
3 \\
4 \\
5\end{array}$ & $\begin{array}{l}19 \\
29 \\
29 \\
29 \\
28\end{array}$ & $\begin{array}{l}25 \\
25 \\
23 \\
26 \\
23\end{array}$ & $\begin{array}{l}24 \\
25 \\
24 \\
25 \\
22\end{array}$ & $\begin{array}{l}31 \\
20 \\
23 \\
19 \\
26\end{array}$ \\
\hline$M$ & & $26.8(3.92)$ & $24.4(1.20)$ & $24.0(1.10)$ & $23.8(4.35)$ \\
\hline M 10 & $\begin{array}{l}1 \\
2 \\
3 \\
4 \\
5\end{array}$ & $\begin{array}{l}32 \\
16 \\
21 \\
31 \\
28\end{array}$ & $\begin{array}{l}21 \\
30 \\
26 \\
23 \\
20\end{array}$ & $\begin{array}{l}22 \\
30 \\
25 \\
23 \\
20\end{array}$ & $\begin{array}{l}24 \\
23 \\
27 \\
22 \\
31\end{array}$ \\
\hline$M$ & & $25.6(6.15)$ & $24.0(3.63)$ & $24.0(3.41)$ & $25.4(3.26)$ \\
\hline
\end{tabular}

Note-Mean transitions (with standard deviations) are also provided. 
Table 3

Wheel and Sucrose Reinforcers Obtained During the Last Five Sessions

\begin{tabular}{cccccc} 
Rat & Wheel & Sucrose & Total & Wheel & Sucrose \\
\hline H3 & 247 & 253 & 15 & 7 & 8 \\
H4 & 232 & 268 & 10 & 7 & 3 \\
H8 & 250 & 250 & 88 & 86 & 2 \\
H9 & 246 & 254 & 20 & 17 & 3 \\
H11 & 248 & 252 & 15 & 14 & 1 \\
H12 & 241 & 259 & 18 & 18 & 0 \\
M2 & 242 & 258 & 30 & 18 & 12 \\
M7 & 244 & 256 & 26 & 22 & 4 \\
M9 & 258 & 242 & 50 & 43 & 7 \\
M10 & 251 & 249 & 48 & 47 & 1 \\
$M$ & 245.9 & 254.1 & 32.0 & 27.9 & 4.1 \\
\hline
\end{tabular}

Note--In addition, total reinforcers obtained following a single leverpress are broken down by type of upcoming reinforcer signaled.

Figure 4 shows leverpressing rates broken down by previous (wheel, sucrose) and upcoming (wheel, sucrose) reinforcer combinations for each animal. In general, leverpressing rates in the presence of the sucrose stimulus, regardless of prior reinforcer type, were greater than in the presence of the wheel stimulus, regardless of prior reinforcer type. Most, but not all, rats showed this pattern. For example, Rats H11 and H12 showed comparable response rates for the wheel-wheel, wheel-sucrose, and sucrose-sucrose combinations, but lower rates for the sucrose-wheel combination. For the group, mean leverpressing rates for the wheel-wheel, wheel-sucrose, sucrose-wheel, and sucrose-sucrose combinations were $41.84,62.63,37.00$, and $60.61 \mathrm{presses} / \mathrm{min}$, respectively. A two-way repeated measures ANOVA with previous reinforcer type (wheel, sucrose) and upcoming reinforcer type (wheel, sucrose) as within-subjects variables revealed a significant effect of upcoming reinforcer type $[F(1,9)=44.32, p<.001]$, but no effect of previous reinforcer $[F(1,9)=3.59, p>.05]$ or previous $\times$ upcoming reinforcer interaction $[F(1,9)=1.10, p>.05]$.

Figure 5 shows median PRP durations as a function of combinations of previous and upcoming reinforcer types. In contrast to response rates, a consistent pattern is much less readily apparent. Several rats, most notably $\mathrm{H} 3, \mathrm{H} 8, \mathrm{H} 9, \mathrm{H} 11, \mathrm{M} 9$, and M10 show shorter PRPs for the wheel-sucrose and sucrose-sucrose combinations than for the wheel-wheel and sucrose-wheel combinations. The remaining rats show varied patterns of PRPs. For the group, average median PRPs for the wheel-wheel, wheelsucrose, sucrose-wheel, and sucrose-sucrose combinations were $18.92,13.90,16.23$, and $12.54 \mathrm{sec}$, respectively. A two-way repeated measures ANOVA with previous reinforcer type (wheel, sucrose) and upcoming reinforcer type (wheel, sucrose) as within-subjects variables revealed a significant effect of upcoming reinforcer type $[F(1,9)=10.90, p<.01]$, but no effect of previous reinforcer $[F(1,9)=2.56, p>.05]$ or previous $\times$ upcoming reinforcer interaction $[F(1,9)=2.43, p>.05]$.

Finally, Table 4 shows the mean number of revolutions per session as well as mean revolutions within successive
5 -sec segments of the reinforcer interval. On average, rats made 415.4 revolutions over 49.18 reinforcers. Stated as a wheel-running rate, the mean running rate was 33.75 revolutions/min. With respect to the pattern of running within the reinforcement period, for all animals, the highest rate of running occurred during the second $5-\mathrm{sec}$ segment rather than the first or the last. Mean revolutions during the first, second, and final segments were 135.9, 159.6, and 119.9 revolutions, respectively. The lower number of revolutions during the first 5 -sec segment most likely reflects the time required for the animal to commence running. The lower running rate during the third segment may reflect either fatigue or a temporal discrimination of the reinforcer interval.

\section{DISCUSSION}

In the present study, rats learned to discriminate between stimuli predictive of qualitatively different reinforcers. The evidence for this discrimination was that, on FI schedules, rats responded sooner and at a higher rate in the presence of a stimulus predictive of sucrose reinforcement than in the presence of a stimulus predictive of wheel-running reinforcement. Analysis of local response rates and PRP durations broken down by the type of the immediately prior reinforcer and the type of the upcoming reinforcer signaled by the stimulus revealed no effect of the previous reinforcer type. Instead, differences in responding and pausing were directly related to the stimulus signaling the upcoming reinforcer. This analysis suggests that, for this pair of values of these reinforcers, excitatory stimulus effects rather than inhibitory after-effects account for differences in response rates.

The particular importance of these results is that they imply that the difference in response rate asymptotes observed by Belke (1998) for the same reinforcers used in the present study was a function of differences in the efficacy of these reinforcers for generating responding rather than differences in satiation or fatigue. Additional evidence consistent with this conclusion comes from research suggesting that fatigue does not contribute substantially to long pauses following wheel running. Belke (2000) showed that when the duration of the opportunity to run as a reinforcing consequence varied within a session, the number of revolutions that occurred prior to a pause accounted for less than $10 \%$ of the variance in pause duration. Furthermore, the observed relation was largely due to shorter PRP durations following a small number of revolutions (i.e., fewer than 10 ) with no systematic increase in pause duration with further increments in revolutions run. The lack of a strong relationship between revolutions and pause duration is consistent with the present data, which suggest, for the limited parameters investigated, that inhibitory after-effects do not play a substantial role in determining pause duration.

If this is the case, then the results of the present study, in conjunction with Belke's (1998) findings, contribute to a growing body of research that question the validity of 


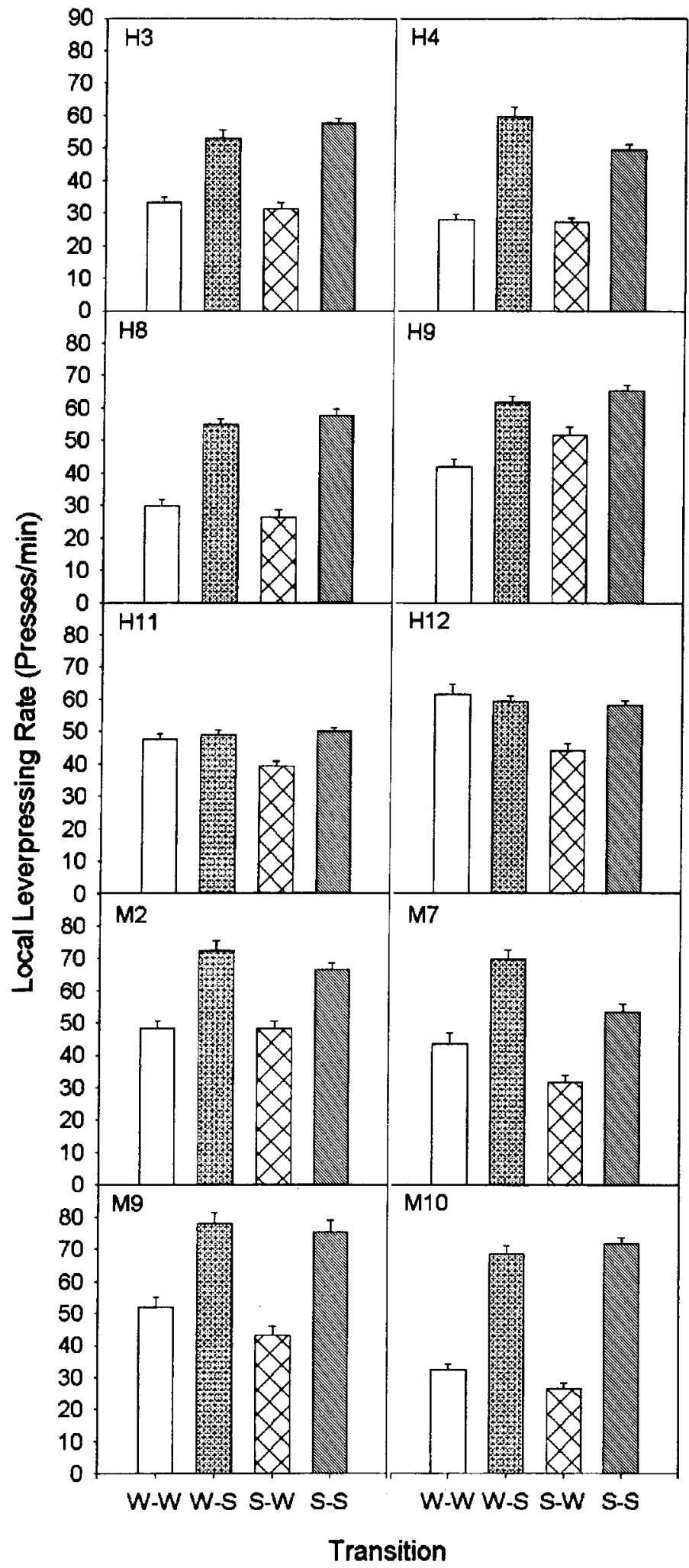

Figure 4. Mean local leverpressing rates (presses/min) as a function of transition type for each animal, including M2 to M10. Transitions were defined by the type of the immediately previous reinforcer and the stimulus signaling the type of the upcoming reinforcer. Wheel-running and sucrose reinforcers are denoted with a "W" and an "S," respectively. Standard errors are depicted for each mean. 


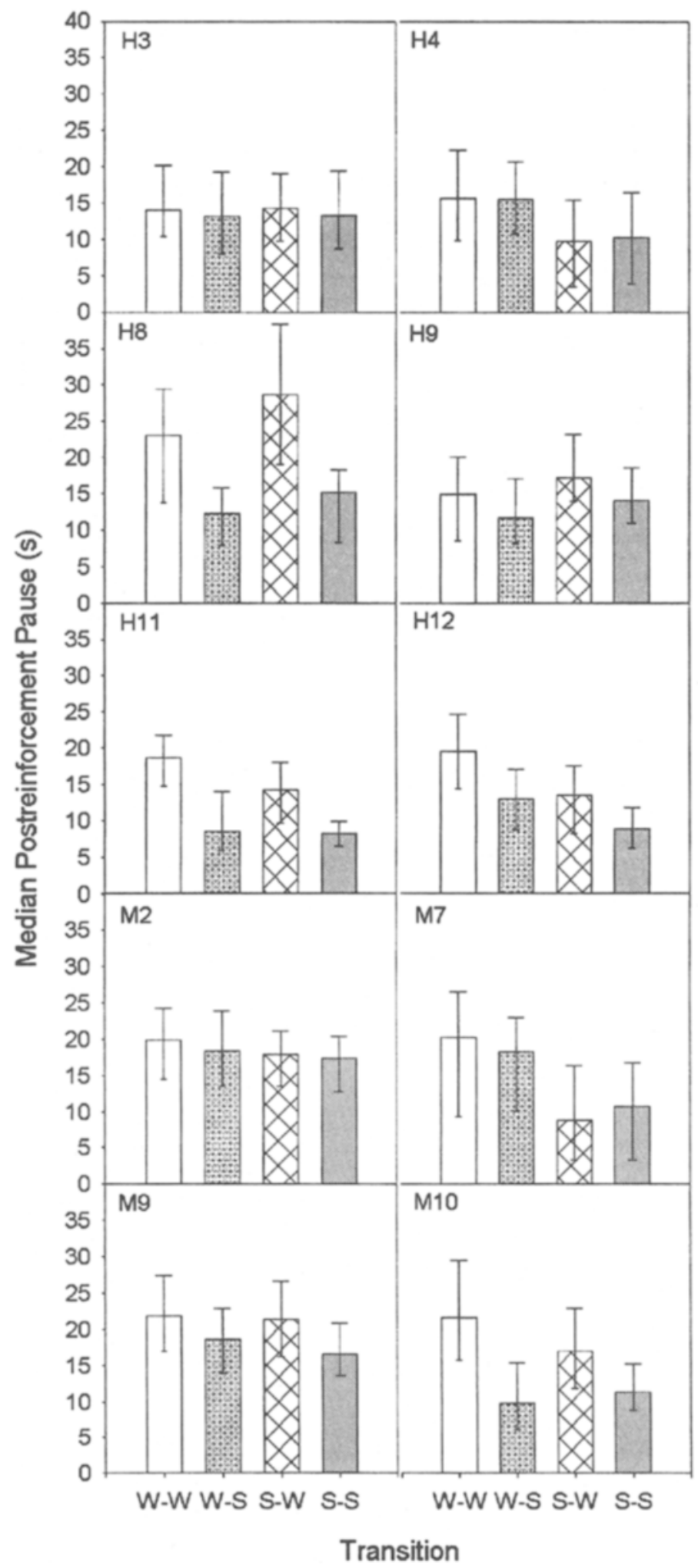

Figure 5. Median postreinforcement pause duration (sec) as a function of transition type for each animal, including M2 to M10. Transitions were defined by the type of the immediately previous reinforcer and the stimulus signaling the type of the upcoming reinforcer. Wheel-running and sucrose reinforcers are denoted with a "W" and an "S," respectively. Interquartile ranges are depicted for each median. 
Table 4

Mean Revolutions/Session, Standard Deviations, Wheel-Running Reinforcers, and Revolutions During the First, Second, and Final 5-Sec Segments of the 15-Sec Reinforcement Period for Each Animal Over the Final Five Sessions

\begin{tabular}{ccccrrr}
\hline & \multicolumn{2}{c}{ Revolutions } & & & & \\
\cline { 2 - 3 } Rat & $M$ & $S D$ & Reinforcers & $0-5$ & $6-10$ & $11-15$ \\
\hline H3 & 370.6 & 41.33 & 49.40 & 92.6 & 149.6 & 128.4 \\
H4 & 550.2 & 46.77 & 46.40 & 200.0 & 203.0 & 147.2 \\
H8 & 334.4 & 76.86 & 50.00 & 99.4 & 121.8 & 113.2 \\
H9 & 364.4 & 28.20 & 49.20 & 121.2 & 142.0 & 101.2 \\
H11 & 516.2 & 49.60 & 49.60 & 186.8 & 194.6 & 134.6 \\
H12 & 519.0 & 58.82 & 48.20 & 176.6 & 207.0 & 135.4 \\
M2 & 360.8 & 51.91 & 48.40 & 108.8 & 148.4 & 103.6 \\
M7 & 323.2 & 25.23 & 48.80 & 101.2 & 121.6 & 100.6 \\
M9 & 477.6 & 63.99 & 51.60 & 172.4 & 182.0 & 123.2 \\
M10 & 337.4 & 39.44 & 50.20 & 99.8 & 126.0 & 111.6 \\
$M$ & 415.4 & & 49.18 & 135.9 & 159.6 & 119.9 \\
\hline
\end{tabular}

Herrnstein's (1970) matching law equation as an account of single alternative responding. Instead, these results support alternative models, such as linear systems theory (McDowell \& Wood, 1984), that predict differences in response rate asymptotes as a function of differences in reinforcer magnitude.

It is also important to note the limitations of the present study. The results speak to the relative roles of excitatory stimulus effects and inhibitory after-effects associated with the pair of reinforcer values that generated differences in response rate asymptotes. They do not inform us about the relative roles of these effects across differing combinations of sucrose concentrations, wheel-running reinforcer durations, and reinforcement schedule values. Parametric assessments of different combinations of these variables are needed to provide a more complete understanding of the roles played by these effects on responding maintained by sucrose and running.

\section{REFERENCES}

BELKE, T. W. (1998). Qualitatively different reinforcers and parameters of Herrnstein's (1970) response-strength equation. Animal Learning \& Behavior, 26, 235-242.

BELKE, T. W. (2000). Varying wheel-running reinforcer duration within a session: Effect on the revolution-postreinforcement pause relation. Journal of the Experimental Analysis of Behavior, 73, 225-239.

Bradshaw, C. M., Ruddle, H. V., \& SzaBadi, E. (1981). Relationship between response rate and reinforcement frequency in variableinterval schedules: II. Effect of the volume of sucrose reinforcement. Journal of the Experimental Analysis of Behavior, 35, 263-269.

Bradshaw, C. M., Szabadi, E., Ruddle, H. V., \& Pears, E. (1983). Herrnstein's equation: Effect of deprivation level on performance in variable-interval schedules. Behavior Analysis Letters, 3, 267-273.

ConRaD, D. G., \& Sidman, M. (1956). Sucrose concentration as reinforcement for leverpressing by monkeys. Psychological Reports, 2 , 381-384.

Dallery, J., McDowell, J. J., \& LANCASTer, J. S. (2000). Falsification of matching theory's account of single-alternative responding: $k$ varies with sucrose concentration. Journal of the Experimental Analysis of Behavior, 73, 23-43.

DE Villiers P., \& Herrnstein, R. J. (1976). Toward a law of response strength. Psychological Bulletin, 83, 1131-1153.

GutTMAN, N. (1954). Equal reinforcement values for sucrose and glucose solutions compared with equal-sweetness values. Journal of Comparative \& Physiological Psychology, 47, 358-361.

HERRNSTEIN, R. J. (1970). On the law of effect. Journal of the Experimental Analysis of Behavior, 13, 243-266.

HERRNSTEIN, R. J. (1974). Formal properties of the matching law. Journal of the Experimental Analysis of Behavior, 21, 159-164.

Heyman, G. M., \& Monaghan, M. M. (1987). Effects of changes in response requirement and deprivation on the parameters of the matching law equation: New data and review. Journal of Experimental Psycholzogy: Animal Behavior Processes, 13, 384-394.

Heyman, G. M., \& Monaghan, M. M. (1994). Reinforcer magnitude (sucrose concentration) and the matching law theory of response strength. Journal of the Experimental Analysis of Behavior, 61, 505516.

KRAELING, D. (1961). Analysis of amount of reward as a variable in learning. Journal of Comparative \& Physiological Psychology, 54, 560-565.

MCDOWELL, J. J., \& DALlery, J. (1999), Falsification of matching theory: Changes in the asymptote of Herrnstein's hyperbola as a function of water deprivation. Journal of the Experimental Analysis of Behavior, 72, 251-268.

MCDOWELL, J. J., \& Wood, H. M. (1984). Confirmation of linear system theory prediction: Changes in Herrnstein's $k$ as a function of changes in reinforcer magnitude. Journal of the Experimental Analysis of Behavior, 41, 183-192.

Perone, M., \& Courtney, K. (1992). Fixed-ratio pausing: Joint effects of past reinforcer magnitude and stimuli correlated with upcoming magnitude. Journal of the Experimental Analysis of Behavior, 57, 33 46.

(Manuscript received April 6, 2000; revision accepted for publication July 20,2000 .) 